\title{
COVID-19 pneumonia-residual changes on CT scan are they all fibrosis
}

Anirudh Kohli

Breach Candy Hospital Trust, Mumbai, Maharashtra, India. E-mail: dranirudhkohli@gmail.com

COVID-19 pneumonia causes diffuse alveolar damage. This passes through three stages, an initial exudative phase where there is minimal exudation of fluid
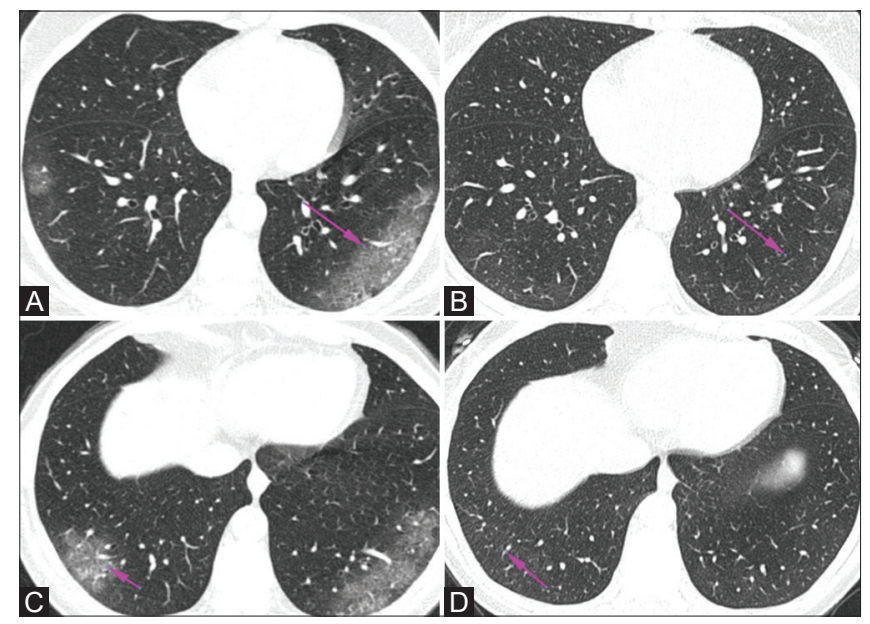

Figure 1 (A-D): HRCT (A and B) Demonstrates typical ground-glass densities in a subpleural location with a prominent vessel in the ground-glass densities. Follow up HRCT after 10 days (C and D) reveals total clearing of COVID-19 pneumonia into the alveoli. As a result, imaging appearances will be negative or subtle abnormalities off ground glass will be seen. Subsequently, with increased

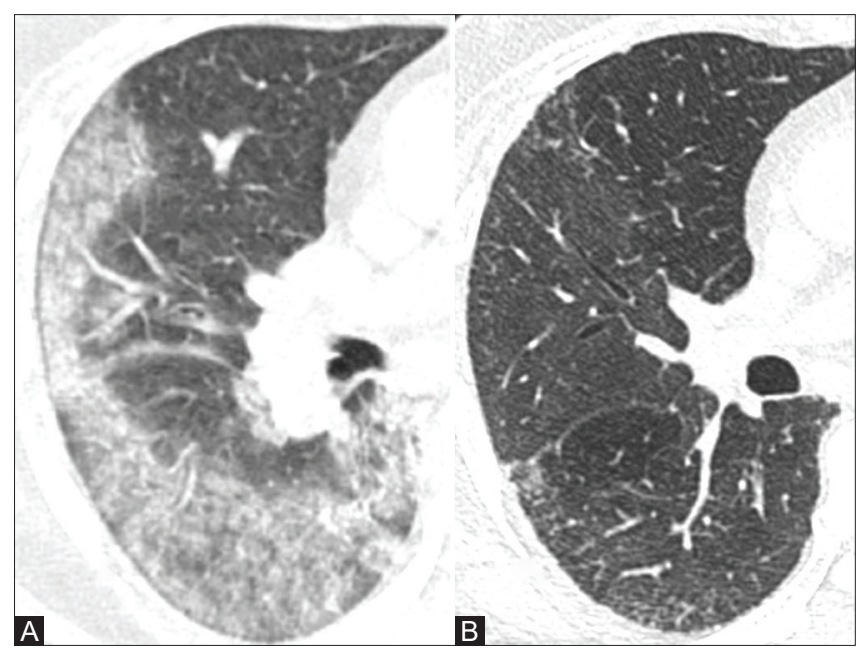

Figure 2 (A and B): HRCT (A) demonstrates ground-glass densities/ consolidations in subpleural region. Follow up CT (B) after 15 days reveals total clearing of abnormalities

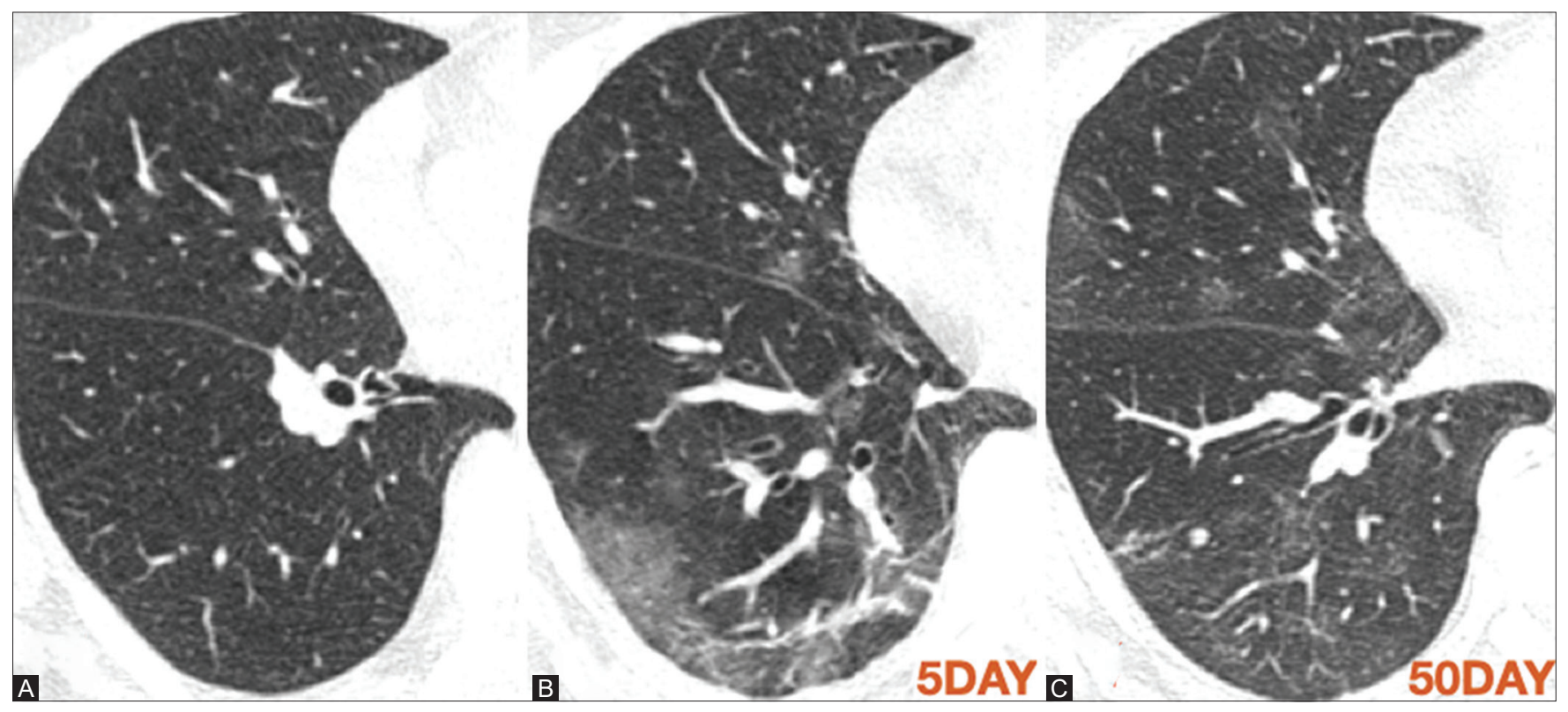

Figure 3 (A-C): (A) HRCT early in disease reveals no abnormality (stage I). (B) HRCT later 5 days liver reveals typical subpleural ground-glass densities with reticular bands. Follow up HRCT (C) on day 50 from first scan reveals clearing of opacities with few residual ground-glass densities 


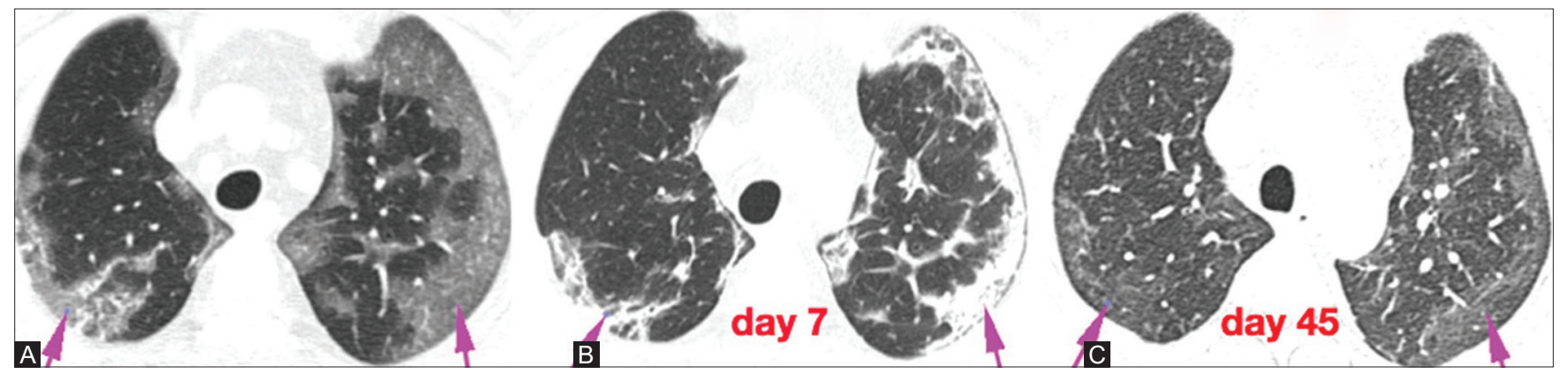

Figure 4 (A-C): HRCT (A) reveals typical subpleural ground-glass densities. HRCT (B) after 7 days of first scan reveals transition of ground-glass densities to dense consolidation with reticular opacities. HRCT (C) 45 days after first scan reveals nearly total resolution with minimal ground-glass densities

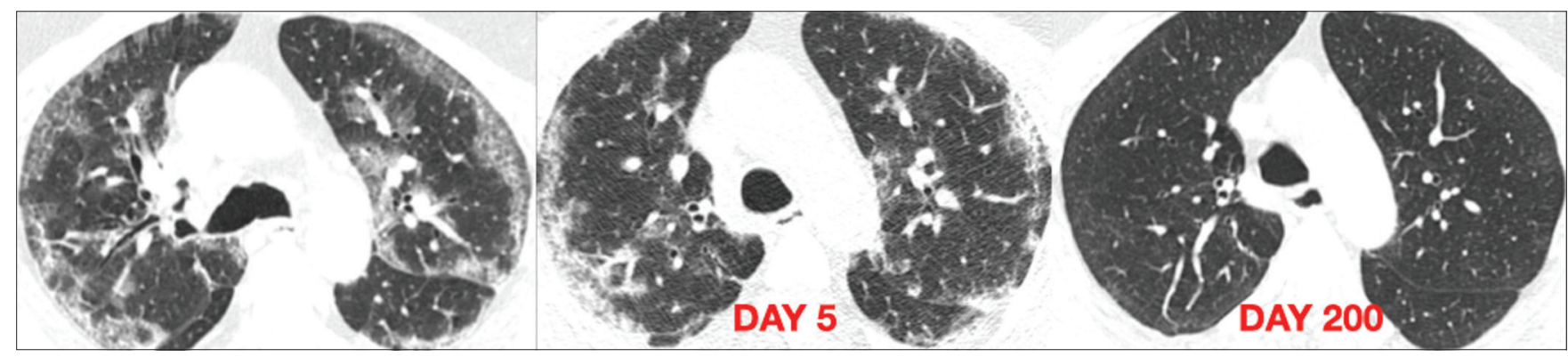

Figure 5 (A-C): HRCT (A) reveals typical peripheral subpleural ground-glass densities as well as peribronchovascular densities. HRCT (B) after 5 days reveals evolution of ground-glass densities to consolidation and reticular abnormalities. HRCT (C) 200 days after first scan reveals clearing of all abnormalities

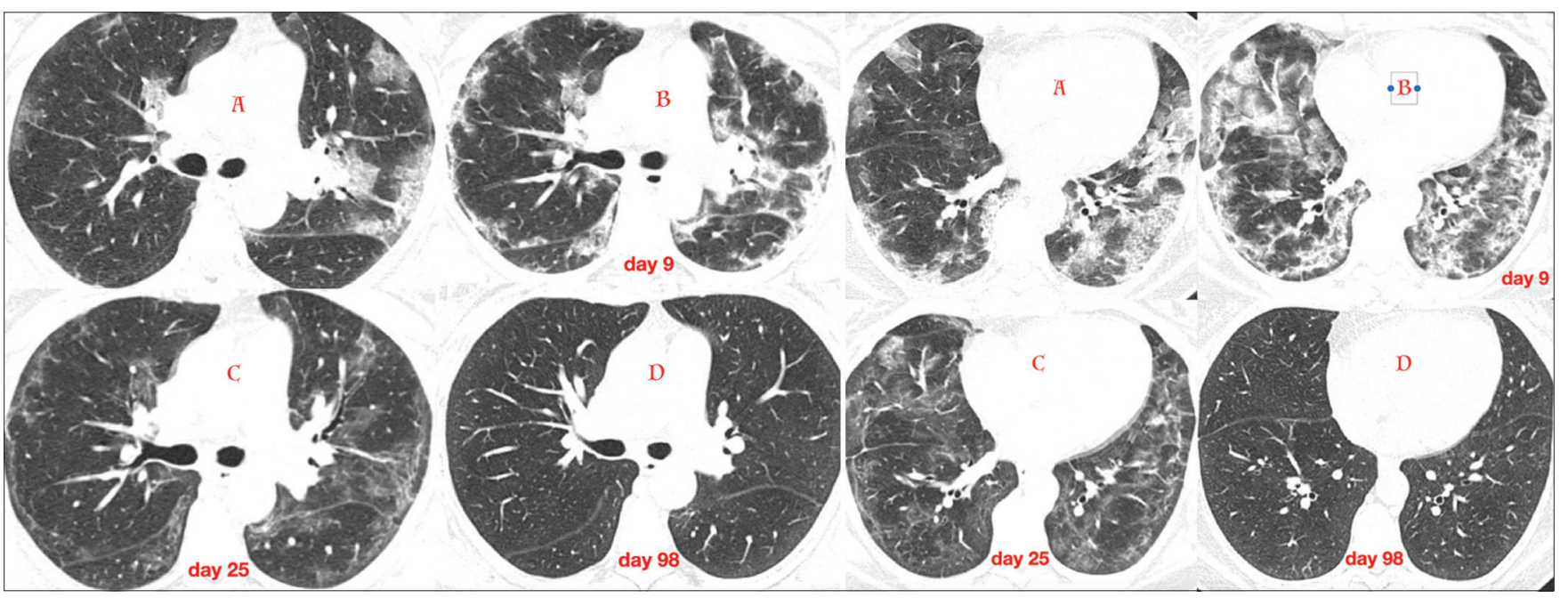

Figure 6 (A-D): HRCT (A) typical peripheral ground-glass and consolidations in subpleural regions. HRCT (B) 9 days after first scan evolve into consolidations, reticular abnormalities resembling organising pneumonia. HRCT (C) 25 days after first scan consolidations resolved with minimal ground-glass densities and subpleural curvilinear lines. HRCT (D) CT study at day 98 from first scan reveals total clearing of all abnormalities also subpleural curvilinear lines which is often reported as fibrosis

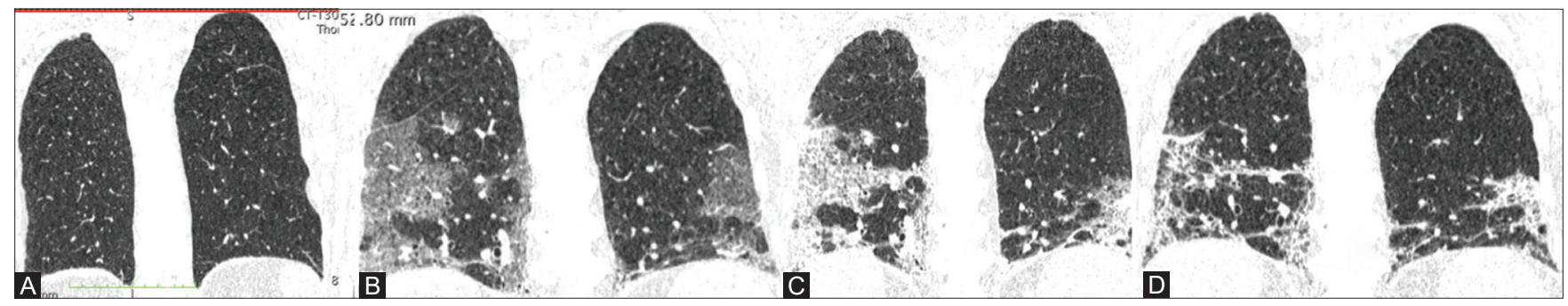

Figure 7 (A-D): Series of HRCT images in coronal plane demonstrating the evolution of COVID-19 pneumonia. HRCT (A) normal, HRCT (B) ground-glass densities, HRCT (C) consolidations with septal thickenings, HRCT (D) residual reticular opacities of organising pneumonia which with time should resolve 


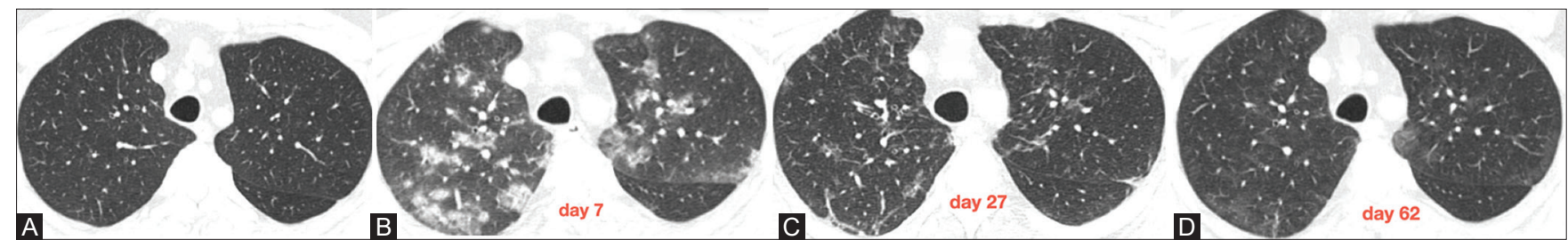

Figure 8 (A-D): HRCT (A) early in disease reveals no abnormality. HRCT (B) 7 days after first scan reveals peribronchovascular ill-defined consolidations. HRCT (C) 27 days after first scan demonstrate resolution of the consolidations. HRCT (D) 62 days after first scan shows near total resolution

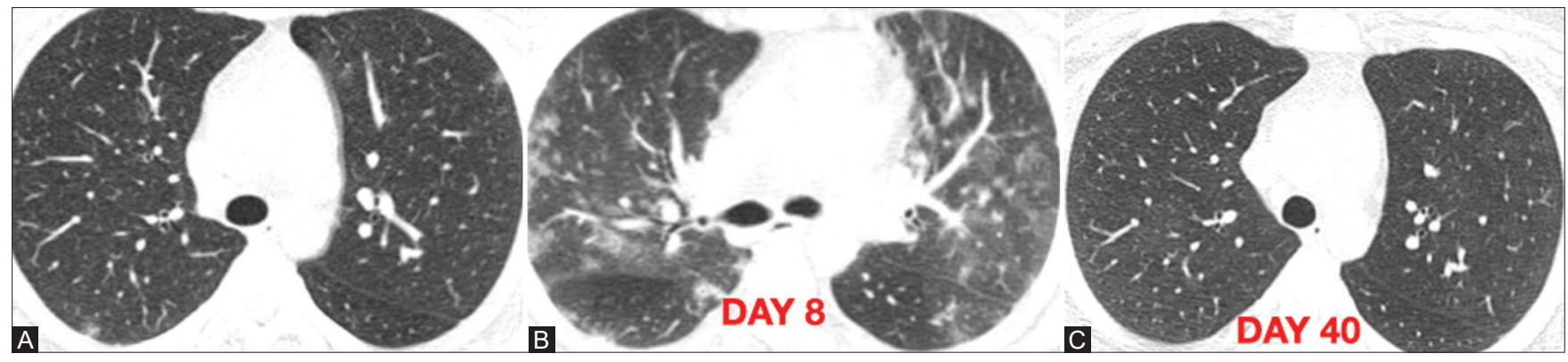

Figure 9 (A-C): HRCT (A) demonstrates subtle ground-glass densities in right posterior subpleural region of lower lobe. HRCT (B) 8 days later, COVID-19 pneumonia evolves with subpleural and peribronchovascular ground-glass densities and consolidations. HRCT (C) 40 days after first scan reveals total resolution of abnormalities

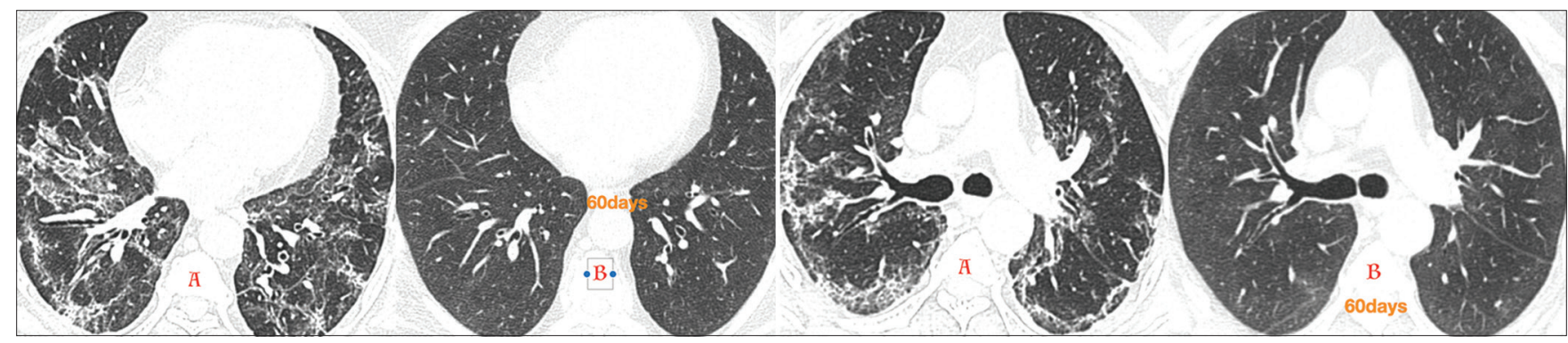

Figure 10 (A and B): HRCT (A) demonstrates subpleural and peribronchovascular ground-glass, consolidations and reticular abnormalities which totally resolved in HRCT (B) done after 60 days

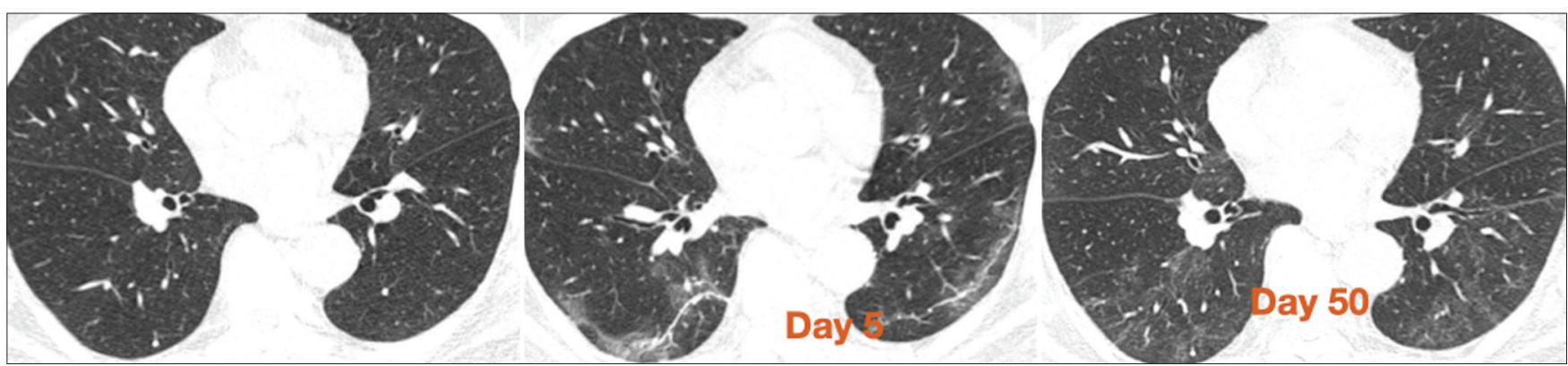

Figure 11: HRCT (A) COVID-19 pneumonia in resolving phase with subpleural curvilinear lines often termed fibrosis this on follow up scan shows total resolution

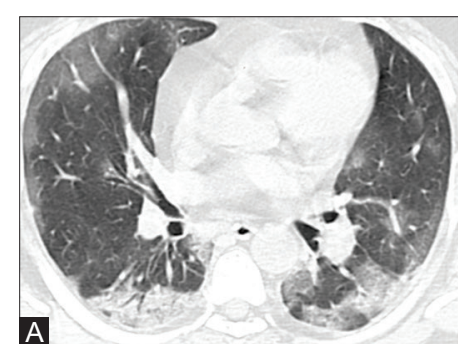

Figure 12 (A-D): HRCT (A) demonstrates typical COVID-19 pneumonia in inflammatory stage with ground-glass densities/consolidations in subpleura location. HRCT (B) after 11 days progressed to fibro proliferative phase with ground-glass densities transiting to organising pneumonia HRCT (C) after 45 days organising pneumonia clears with residual ground-glass densities. HRCT (D) after 112 days shows resolution of all abnormalities 


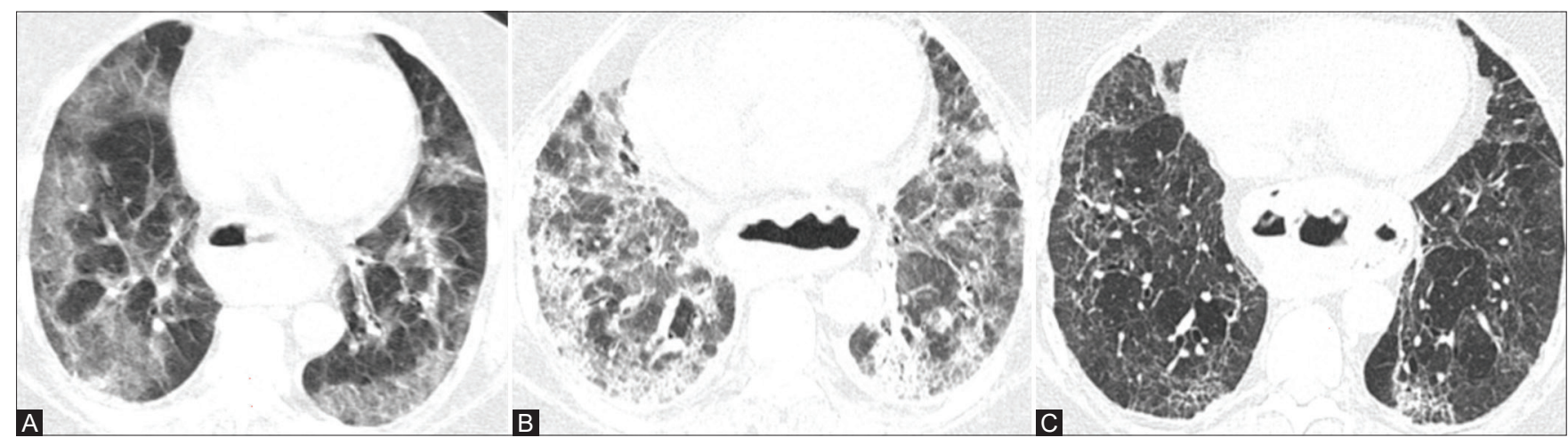

Figure 13 (A-C): HRCT (A) shows typical COVID19 pneumonia with subpleural distribution of ground glass densities. HRCT (B) after 30 days shows ground glass densities turning into extensive consolidation and reticular abnormalities HRCT (C) after 155 days shows considerable resolution

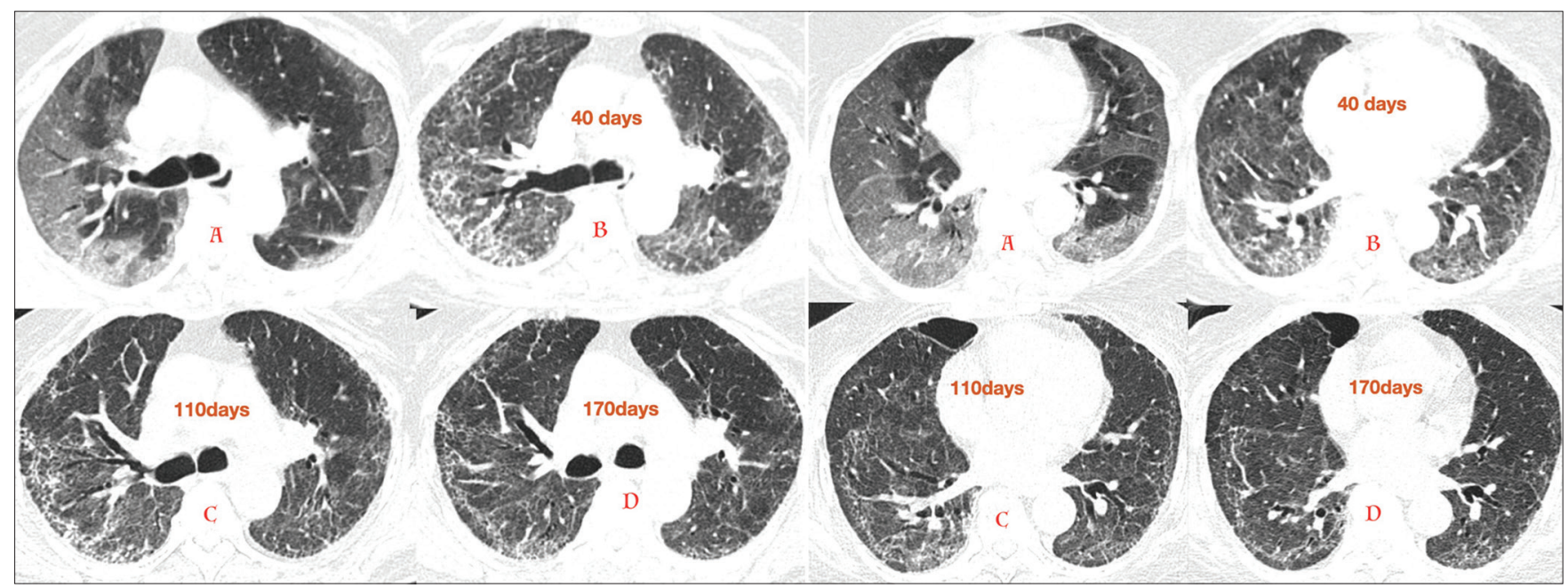

Figure 14 (A-D): HRCT (A) Reveals subpleural ground glass densities typical of COVID 19 pneumonia Follow up study after 40 days (B) reveals subpleural and peribronchovascular interstial thickening which shows mild regression on follow up scans done 110 (C) and 170 days (D) post first scan. Persistence of these findings after 6 months raises the possibility of true fibrosis though further follow up will be useful as there appears to be a mild persistent regression

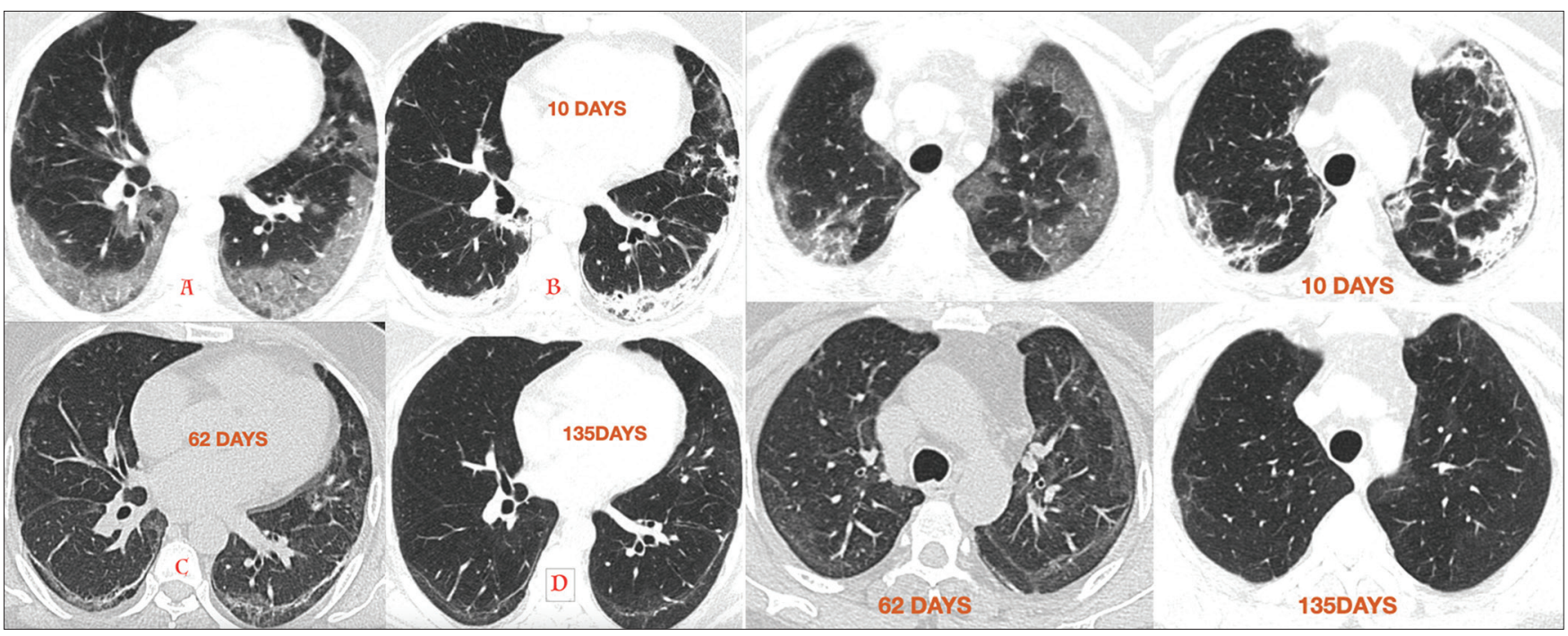

Figure 15: HRCT (A) revels subpleural ground glass of covid pnuemonia Follow up after 10 days reveals ground glass has evolved to consolidation in subpleural regions which further resolves to subpleural curvilinear line on HRCT (C) done after 62 days of first scan. Final scan (D) after 135 days reveals persistence of subpleural curvilinear line 
exudation into the alveoli it passes into the next stage, an inflammatory stage. Depending on the extent of alveolar exudation the appearances on imaging are ground glass densities, consolidation or a combination of these. Finally, the diffuse alveolar damage passes into a reparative phase where there is proliferation of epithelial cells and fibroblasts with collagen deposition. On imaging the appearances in this stage are off an organising pneumonia. The brunt of the disease process of COVID-19 is in the interstium, thus in the peripheral subpleural and peribronchovascular regions. As the organising pneumonia clears, there are reticular opacities in the subpleural and peribronchial regions. Often there is a subpleural curvilinear line and residual peribronchovascular and subpleural reticular abnormalities which resemble fibrosis seen in ILD. This is the main consideration, are these to be reported as fibrosis? this is important as fibrosis is irreversible. However, with time sometimes even 4-6 months later most of these abnormalities clear up, even the subpleural curvilinear lines. Only very few cases of persistent reticular abnormalities have been seen. Time will tell whether these are also really fibrosis or slow resolving organising pneumonia since these may clear with time.
To call these fibrosis may be a bit premature.

Appended are a sampling of cases demonstrating progression and regression patterns in COVID-19 pneumonia.

This is an open access journal, and articles are distributed under the terms of the Creative Commons Attribution-NonCommercial-ShareAlike 4.0 License, which allows others to remix, tweak, and build upon the work non-commercially, as long as appropriate credit is given and the new creations are licensed under the identical terms.

\begin{tabular}{|l|l|}
\hline \multicolumn{2}{|c|}{ Access this article online } \\
\hline Quick Response Code: & Website: \\
\hline & www.ijri.org \\
\cline { 2 - 3 } & DOI: \\
\hline
\end{tabular}

Cite this article as: Kohli A. COVID-19 pneumonia-residual changes on CT scan are they all fibrosis. Indian J Radiol Imaging 2020;30:415-9.

Received: 03-Jan-2020 Accepted: 03-Jan-2020 Published: 13-Jan-2021 